\title{
Cidades Inteligentes e City Information Modeling
} Smart Cities and City Information Modeling

\author{
Arivaldo Leão de Amorim \\ LCAD | Faculdade de Arquitetura | UFBA, Brasil \\ alamorim@ufba.br
}

\begin{abstract}
This paper presents and discusses the relationship between the concepts of Smart Cities and City Information Modeling (CIM). It conveys the notion that these are complementary and not competing concepts, as one might think at first glance. On the other hand, the paper demonstrates the importance of these concepts to overcome the challenges to the cities of the $21^{\text {st }}$ century, from findings contained in official documents published by the United Nations (UN), to analyze the growth of world population and the emergence of new cities to house population groups. Finally, this paper argues that the CIM with an inducing factor for the Smart City is an important resource to help improve the quality of life in cities.
\end{abstract}

Keywords: Smart Cities; City Information Modeling; Sustainability; Cities of the Future; Information Modeling

\section{Os desafios das cidades do século $\mathbf{X X I}$}

Este trabalho apresenta e discute a relação entre os conceitos de Cidades Inteligentes e City Information Modeling (CIM), mostrando que estes são conceitos complementares e não concorrentes, como se poderia pensar à primeira vista. Por outro lado, o artigo busca mostrar a importância desses conceitos frente aos desafios colocados para as cidades do século XXI, a partir de constatações contidas em documentos oficiais publicados pela Organização das Nações Unidas (ONU), que analisam o crescimento da população mundial e o surgimento de novas cidades para abrigar os contingentes populacionais.

Segundo dados do Fundo de População das Nações Unidas (FNUAP), a população mundial atingiu 7,2 bilhões de habitantes em 2013. Embora a taxa de crescimento populacional venha apresentado redução ano a ano, a estimativa é que em 2050 a população do planeta seja 9 bilhões de habitantes, crescendo à taxa de $0,33 \%$ ao ano, bem inferior à taxa atual de crescimento que é 2,02\% (ONU, 2014), mas ainda assim projeta-se uma população da ordem de 11 bilhões de pessoas para o final do século XXI.

Ainda segundo a ONU (2014), estima-se que a população das regiões desenvolvidas permanecerá praticamente inalterada em torno de 1,3 bilhão de pessoas até 2050 . Entretanto, nos 49 países menos desenvolvidos a população deverá passar dos atuais 900 milhões de habitantes para 1,8 bilhões, no mesmo período.

No caso brasileiro as perspectivas não são mais animadoras. A população atual da ordem de 207 milhões de habitantes cairá para 200 milhões no ano de 2100 , mas ainda atingirá o pico de 238 milhões de habitantes em 2050, quando a população deverá começar a decrescer em função das taxas de natalidade mais baixas. Esses números indicam que no prazo de 35 anos precisarão ser construídas moradias para 31 milhões de pessoas, e nos 50 anos seguintes as moradias usadas por 38 milhões de brasileiros ficarão ociosas.

Confirmados esses números, e considerando o déficit quantitativo e qualitativo de habitações atualmente existente no Brasil, o quadro que se apresenta é preocupante, pois além dos problemas habitacionais somam-se as questões da deficiência da infraestrutura nacional como um todo. Assim, a superação dessas condições francamente desfavoráveis constitui um grande desafio a ser superado pelos brasileiros até o final do século XXI.

Ainda segundo o relatório (ONU, 2014), em 1990 as chamadas megacidades, ou seja, aquelas com a população acima de 10 milhões de habitantes eram apenas 10, distribuídas por todo o mundo. Em 2014, apenas 24 anos depois, estas cidades já eram 21, e projeta-se que no ano de 2030 elas serão 41 cidades. Em relação às cidades entre $5 \mathrm{e}$ 10 milhões de habitantes, elas passarão de 43 em 2014 para 63 em 2030. E, finalmente, as cidades entre 1 e 5 milhões de habitantes, que eram 417 em 2014, são estimadas em 558 para o horizonte de 2030.

Partindo-se de um raciocínio simplista e conservador dentro das projeções apontadas, num período de 16 anos (2014 2030) deverão ser construídas em escala mundial, 20 cidades para 10 milhões de habitantes, 20 cidades para 5 milhões de habitantes, e 171 cidades para mais de 1 milhão de habitantes, sem contar com a necessidade de milhares de pequenas e médias cidades.

Não bastasse as demandas para a construção do novo visando 0 atendimento do crescimento populacional, concomitantemente se observa que a maior parte das cidades brasileiras e do mundo não possuem a infraestrutura necessária para alcançar os padrões de desenvolvimento 
sustentável estabelecidos pela Agenda 2030 para o Desenvolvimento Sustentável, proposta pela ONU.

Verifica-se ainda, que mesmo nas grandes cidades onde a infraestrutura urbana pode ser reconhecida como adequada, na maioria desses assentamentos os sistemas, as instalações e os recursos estão envelhecidos, necessitando de ampliações, modernizações etc.

Por outro lado, faz-se notar a crescente complexidade urbana, onde os profissionais que trabalham com os sistemas de infraestrutura da cidade e os serviços públicos em geral demandam novos recursos para suprir as necessidades no planejamento, no projeto, na construção, na gestão dos ativos urbanos, bem como na renovação dos mesmos.

Assim, o desenvolvimento de tecnologias, sistemas, ferramentas, modelos e padrões têm sido propostos e implementados para fazer frente às demandas das populações urbanas por novos serviços, a expansão ou a melhoria dos sistemas existentes, sempre buscando a redução dos custos e assegurando padrões de qualidade de vida para todos os cidadãos.

$O$ quadro aqui descrito ressalta os desafios que estão postos para as cidades do século XXI são de grande envergadura, demandando novos paradigmas e novas abordagens para 0 atendimento das novas necessidades, bem como do passivo acumulado, buscando a consequente melhoria da qualidade de vida e a almejada justiça social.

Por fim, este trabalho procura mostrar o City Information Modeling (CIM) com um fator indutor para a estruturação das Cidades Inteligentes, e por conseguinte, um importante recurso para auxiliar a melhoria da qualidade de vida nas cidades.

\section{Planejando as cidades do futuro}

O cenário esboçado na seção anterior não é nada otimista. $O$ crescimento propulacional previsto para os próximos anos virá acompanhado do crescimento da pobreza, da violência, da poluição, da crise da água, dos alimentos e da degradação ambiental. De forma a superar ou mesmo amenizar os desafios que se colocam no horizonte do século $\mathrm{XXI}$, novos caminhos precisam ser encontrados.

Nesse contexto, observa-se que as cidades estão em continua transformação, aumentando a sua complexidade e com demandas sempre crescentes, seja pelo novo, seja pela melhoria daquilo já existente. Com o crescimento populacional o perímetro urbano vai se expandindo, ocupando novas áreas, adensando e verticalizando as já construídas, transformando o uso e a ocupação do solo, e ampliando sua impermeabilização, ou ainda, por meio do aperfeiçoamento ou da implantação novos sistemas de infraestrutura, as cidades vão crescendo, transformando-se, reinventando-se num ciclo de vida dinâmico e complexo.
Os estudos patrocinados pela ONU (2014) mostram que confirmadas as tendência, até 2050 , metade do crescimento da população mundial estará concentrado em nove países: Índia, Nigéria, Paquistão, República Democrática do Congo, Etiópia, Tanzânia, os Estados Unidos, Indonésia e Uganda. À exceção dos Estados Unidos, todos esses países são possuidores de passivos significativos e detentores de pouco desenvolvimento econômico, técnico e científico.

Aqui duas questões se tornam evidentes. Se o atendimento dessas demandas já é um grande desafio para os países desenvolvidos e que possuem os recursos técnicos e econômicos para fazer as adaptações necessárias ao enfrentamento do problema, para os países mais pobres e em desenvolvimento essa tarefa torna-se impossível.

No enfrentamento das questões urbanas, o planejamento, a gestão e o monitoramento compreendem os tradicionais recursos usados pelas administrações públicas para lidar com os problemas relativos à cidade.

Enquanto o planejamento foca na elaboração de estudos, planos, projetos, legislação etc., fica no âmbito da gestão executar o planejamento e as demais ações decorrentes, ou seja, a construção da infraestrutura e das edificações com a implantação, a operação e a manutenção de todos os sistemas e serviços públicos, como a segurança, a saúde e a educação, dentre vários outros. Finalmente, cabe ao monitoramento as funções de controle, de fiscalização e de verificação da qualidade dos serviços públicos ofertados à população e os impactos decorrentes sobre 0 meio ambiente, de modo a realimentar os processos de planejamento e de gestão num contínuo aperfeiçoamento das transformações urbanas (Amorim, 2015).

Todas essas atividades essenciais à vida das cidades foram a partir da segunda metade do século $X X$ se beneficiando paulatinamente dos avanços tecnológicos, e em especial, das tecnologias computacionais para o cumprimento das suas funções.

Assim, foram sendo agregados recursos e ferramentas como o sensoriamento remoto, a aerofotogrametria, 0 imageamento por satélite, e a telemetria dentre outros, visando fornecer dados para o planejamento e os projetos dos assentamentos urbanos e sua infraestrutura, bem como do manejo dos recursos ambientais.

$\mathrm{Na}$ sequência, os conceitos de Computer Aided Design (CAD) e Computer Aided Engineering (CAE), foram incorporados, trazendo ferramentas especializadas para 0 projeto de barragens, canais, vias, pontes e viadutos, túneis, parcelamentos etc. e das próprias edificações, bem como de recursos para a simulação numérica de tráfego, da poluição e do ruído urbano, bem como de modelos para uso do solo e transportes, dentre muitos outros recursos atualmente disponíveis.

Como consequência da junção das tecnologias CAD e dos Sistemas Gerenciadores de Bases de Dados (SGBD), 
surgiram as ferramentas Geographic Information System (GIS), um poderoso recurso para a realização de mapeamentos temáticos e análises espaciais, que foram incorporados aos processos de planejamento, de gestão e de monitoramento das redes de infraestrutura da cidade e das diversas categorias de serviços urbanos.

Por outro lado, a Cartografia Digital baseada em aerofotogrametria supriu o mapeamento sistemático necessário aos processos de planejamento, de projeto, de gestão e de monitoramento das cidades, que rapidamente evoluiu, incorporando recursos como a Modelagem Digital de Terreno (MDT) que permitiu a representação da superfície da terra em três dimensões, e suprindo recursos para as análises do terreno como a drenagem natural, os mapas de declividades, e possibilitando uma melhor avaliação das alterações da superfície terrestre, através de operações de terraplenagem com cálculos precisos dos volumes de terra a serem manipulados, ou mesmo cálculo do volume de água armazenado em barragens, ou ainda, das áreas inundadas, dentre outras aplicações.

Mais recentemente, o paradigma Building Information Modeling (BIM) alterou 0 enfoque até então dado à edificação, que passou a ser objeto de um conjunto integrado de operações sofisticadas ao longo de todo o seu ciclo de vida, que compreendem os mais diversos tipos de simulações numéricas visando a produção de edificações melhores, mais duráveis e mais sustentáveis, através do uso da modelagem procedural que incorpora a geometria tridimensional dos objetos, suas propriedades físicas, comportamentos, relacionamentos, dentre vários outros atributos, em benefício da melhor qualidade do ambiente construído, redução dos impactos ambientais e melhoria da satisfação dos usuários.

Quase que em paralelo, surgem a varredura a laser aerotransportada, a modelagem geométrica e o padrão City Geography Markup Language (CityGML), que agregaram a possibilidade da representação tridimensional da cidade como um todo, incluindo as edificações, as vias, os sistemas de transportes, as redes de infraestruturas, os corpos d'água e a vegetação. Todos esses sistemas, componentes e recursos urbanos são dotados dos seus atributos semânticos mais significativos, que viabilizando um sem número de aplicações GIS em três dimensões, que vão desde os estudos ambientais à paisagem urbana e ao turismo, passando por avaliação da eficiência energética, de ilhas de calor e outras questões relativas ao ambiente construído e antropizado.

Completando o quadro, também surgiram as aplicações da modelagem paramétrica com foco no projeto e no planejamento urbano, naquilo que ultimamente têm sidos denominados de "urbanismo paramétrico", "city $C A D$ " e outras terminologias, para essas ferramentas e recursos. E, mediante essas ferramentas flexíveis e altamente interativas, podem ser realizados estudos e análises da morfologia urbana, e da simulação dos impactos decorrentes da adoção de parâmetros urbanísticos, uso e ocupação do solo, dentre várias outras possibilidades, visando a proposição de ambientes construídos de melhor qualidade, mais eficientes com menores custos de implantação e de manutenção, com redução do consumo de energia e de impactos ambientais.

Dessa forma, com a evolução tecnológica, também foram incorporados uma variedade de sistemas, sensores especializados e câmeras de vídeo, que constituem poderosas redes telemétricas, que vão do monitoramento do tráfego e da segurança pública, à medição da poluição, da precipitação pluviométrica em áreas de risco ou mesmo de simples dados atmosféricos, associados a sistemas de atuação e alarmes em tempo real.

Com a evolução tecnológica, todo esse aparato foi lentamente sendo colocado à disposição dos planejadores, dos projetistas, dos construtores, dos operadores, dos gestores, e dos políticos, e, até mesmo do simples cidadão comum. Assim, a tecnologia foi pervasivamente incorporada aos seus processos cotidianos e a ubiquidade está sendo levada às ultimas consequência com a Internet of Things (loT).

Até então, os recursos tecnológicos aplicados ao planejamento, à gestão e ao monitoramento da cidade deram conta, com maior ou menor eficácia, das necessidades urbanas e das transformações delas decorrentes. Entretanto, desde a virada do século, o contexto que se apresenta é o das megacidades, da superpopulação e da sustentabilidade frente à escassez do solo, da água e da energia, dentre outros recursos naturais. Alia-se a isso, à demanda crescente por novos serviços, a expansão dos padrões de consumo, a incorporação de parcelas das populações que antes não eram atendidas ou estavam marginalizadas, a necessidade do combate à poluição, as mudanças climáticas e a proteção ao meio ambiente. Por fim, somam-se a isso os crescentes custos econômicos e financeiros para o provimento de todas as ações e os serviços necessários para fazer frente a esses desafios.

Nesse sentido, visando o atendimento ou encaminhamento de parte das demandas aqui colocadas, um trabalho particularmente interessante vem sendo desenvolvido pelo Prof. Helmut Bott (s. d.) do Urban Design Institute da Universidade de Stuttgart na Alemanha, naquilo que ele denominou de Digital Planning Systems. A proposta contempla uma abordagem através de recursos tecnológicos em direção ao planejamento e à projetação da cidade sustentável do futuro, onde ele discute uma série de tecnologias, sistemas, ferramentas e novos ambientes de trabalho a serem usados no planejamento e na projetação urbana, a exemplo de:

Ferramentas BIM, como um primeiro passo para a construção da base de dados de informações de projeto e para o equacionamento de outras necessidades, como a demanda anual de energia para aquecimento etc.;

CityCAD, uma ferramenta desenvolvida para o trabalho conceitual nas primeiras fases do planejamento da cidade, onde a estrutura semântica do software permite o ajuste 
automático de todas as informações relevantes, tais como volumes, densidades, estacionamentos, custos, valores, etc., através das alterações dos parâmetros do projeto;

ECOTECT, software para análises e simulações de sombras, reflexões, radiação solar, comportamento térmico, ventilação, acústica, iluminação artificial, fluxos de matérias, e materialidade, tanto nas edificações quanto no ambiente urbano como um todo;

GABI, um programa para a análise de sustentabilidade de fluxos de materiais (insumos e rejeitos) de produtos e processos que ocorrem na cidade e para a cidade;

CadnaA (Computer Aided Noise Abatement), uma ferramenta para cálculo, avaliação, predição e visualização através de gráficos do ruído ambiental;

Fluidyn PANAIR, um software especializado na análise da poluição nas áreas urbanas, levando-se em conta as fontes poluentes como sistemas de transportes, as indústrias etc. e a dispersão atmosférica dos poluentes;

GOSOL, um programa desenvolvido para a análise comparativa e a otimização de energia, inclusive energia solar, visando contribuir para o planejamento e o desenvolvimento urbano;

VISSIM, um software voltado para a simulação microscópica de tráfego, visando a operação multimodal dos sistemas de transportes, e que considera aspectos desde a motorização até os ciclistas e os pedestres;

MATSim (Multi-Agent Transport Simulation), ferramenta que permite a simulação de tráfego baseada em agentes de contextos regionais.

O trabalho do Prof. Bott (s. d.) cita ainda recursos que contemplam:

Ferramentas para simulações de gradientes de temperaturas, propagação de calor, ventilação, turbulência de ar etc., que utilizam recursos de Computational Fluid Dynamics (CFD).

Ou mesmo, novos ambientes de trabalho, como:

ARTHUR (Augmented Round Table for architectural design and urban planning Virtual Reality), ambiente que visa auxiliar o planejamento da cidade através de um "modelo virtual", desenvolvido pela Fraunhofer Institut für Angewandte Informationstechnik, na Alemanha, ou ainda, o

ValueLab, da ETH Zurich, que é um laboratório equipado com seis monitores touch screen de grandes dimensões, interligadas em rede com um computador de alto desempenho, que permite múltiplas simulações através de processamento intensivo em tempo real e executadas em paralelo, possibilitando que os especialistas empreguem métodos de simulações e de análises, para testar diretamente o impacto das suas ideias no projeto em desenvolvimento.

Por fim, o Prof. Bott anuncia como sua meta de pesquisa para os próximos anos é o desenvolvimento de um método integral auxiliado por computador para analisar, monitorar e otimizar o ambiente construído como um todo, através do planejamento e do projeto sustentáveis (Bott, s. d).

Para atender a essas questões aqui colocadas, novos paradigmas e estratégias têm sido propostas e desenvolvidas. Neste artigo serão discutidas duas delas consideradas mais abrangentes: as Cidades Inteligentes e 0 City Information Modeling.

Essas novas abordagens envolvem, sobretudo, racionalidade, diversidade, integração, interação, compartilhamento, sustentabilidade, tecnologias de última geração e uma nova forma de olhar a cidade e o Mundo. Em outras palavras, a compreensão de que todos nós somos responsáveis pelo passivo da humanidade.

\section{Cidades Inteligentes}

O conceito de Smart City, SmarterCity ou "cidade inteligente" é sutil e controverso, existindo uma grande variedade de termos para expressar conceitos semelhantes. No que pese diversos autores terem cunhado diferentes termos dando ênfase neste ou naquele aspecto, no fundo, esses conceitos possuem praticamente o mesmo significado.

A principal ideia contida nele é uso intensivo e extensivo das Tecnologias de Informação e Comunicação (TIC) em todo o seu potencial, visando: aperfeiçoar o desempenho dos "serviços urbanos" de modo a suportar o desenvolvimento econômico, social e cultural; contribuir para o estabelecimento das "indústrias criativas"; propiciar o maior bem estar possível a todos os usuários da cidade, e por fim, assegurar a participação eficaz dos cidadãos em todas as questões que lhe dizem respeito.

Assim, a ideia de cidade inteligente pressupõe fundamentalmente: qualidade de vida para todos os seus moradores e visitantes, e o exercício pleno da cidadania. A adoção de politicas e práticas sustentáveis, com o consumo responsável de materiais e de recursos naturais, ao lado da autossuficiência em energia e em alimentos, bem como a redução de danos ao meio ambiente, fazem parte dessa estratégia.

Nesse sentido, pode-se entender a cidade inteligente como sendo a cidade ideal, um paradigma ainda a ser atingido pela maioria esmagadora das cidades, como mostram Lee e Hancock (2012). Para estes autores, existem no mundo 143 projetos de cidades inteligentes em andamento, onde 47 deles estão localizados na Europa, 40 na Ásia, 35 na América do Norte, 11 na América do Sul e 10 na África e no Médio Oriente. 
Ainda Lee e Hancock (2012) afirmam que na América do Norte e na Europa têm prevalecido projetos que privilegiam a requalificação e a renovação urbana, como os projetos SmartSantander na Espanha e Amsterdam Smart City na Holanda. Enquanto que no Oriente Médio e na Ásia as iniciativas apontam para o planejamento, o projeto e a construção de novas cidades, como Masdar nos Emirados Árabes Unidos e Songdo na Coreia do Sul.

Os números apresentados por Lee e Hancock (2012) mostram o tamanho do desafio a ser superado para a construção de cidades mais sustentáveis e socialmente mais justas, considerando-se que existem no mundo cerca de 38 mil cidades. Levando-se em conta as pequenas aglomerações humanas, este número ultrapassa os 2,5 milhões de assentamentos urbanos. Estas informações foram compiladas em websites e blogs na web. Aqui não está em discussão a precisão das mesmas, mas sim a sua ordem de grandeza para a presente discussão.

Por fim, Finguerut e Fernandes afirmam que "As soluções agrupadas sob a designação de Smart Cities se diferenciam das convencionais, não por possuírem padrões e soluções iguais e replicáveis em qualquer contexto, mas por observarem as necessidades especificas de cada cidade e buscarem soluções que se traduzam em ganhos sociais, econômicos e de qualidade de vida para os cidadãos. Dessa maneira, as cidades são vistas como um aglomerado complexo de sistemas operacionais, ecossistemas e redes de atores, que devem interagir diretamente com um ou mais setores governamentais de maneira participativa, gerando externalidades positivas." (Finguerut e Fernandes, 2014, p. 36).

\section{City Information Modeling (CIM)}

O termo City Information Modeling (CIM) surgiu recentemente na esteira do desenvolvimento e popularização do paradigma Building Information Modeing (BIM), existindo uma conceituação incipiente para o mesmo. Autores como Gil, Beirão, Montenegro e Duarte (2010); Hisham (2010); Gil, Almeida e Duarte (2011), e Stojanovski (2013), dentre vários outros, de certa forma conceituaram o CIM nos seus trabalhos.

Entretanto, observando-se cuidadosamente a abordagem desses autores para City Information Modeling, verifica-se que eles consideram apenas determinados aspectos daquilo que poderia ser considerado como CIM na sua forma mais abrangente, passando ao largo da complexidade envolvida na cidade, seja do ponto de vista dos seus aspectos físicoconstrutivos, seja do ponto de vista dos processos que nela ocorrem.

Ainda autores como Schiefelbein et al. (2015), descrevem o desenvolvimento de um modelo CIM para suportar o gerenciamento de dados e a análise de sistemas prediais de energia dentro de bairros complexos, numa abordagem também muito particular e que envolve sustentabilidade e o retrofit urbano, sem dúvida também uma aplicação importante nesse contexto, mas insuficiente para abarcar as demandas e a complexidade aqui apontada.

Na mesma linha, embora o Prof. Bott (s. d.) nos seus estudos não se refira especificamente ao termo City Information Modeling, o seu trabalho se encaixa perfeitamente nesse contexto, nos aspectos em que se refere ao planejamento e ao projeto dos aspectos físicos e territoriais de uma cidade numa abordagem sistêmica, e que sem dúvida constituem um dos aspectos mais importantes relativos à implementação do CIM.

Sumarizando, constata-se que embora significativas e importantes as contribuições dos autores citados, elas discorrem apenas sobre um subconjunto das questões envolvidas daquilo que deveria ser uma abordagem de City Information Modeling de forma mais abrangente e integral.

Nesse sentido, Amorim (2015) considera que essas definições postas em discussão para o estabelecimento desse novo paradigma, não são suficientemente abrangentes para dar conta da complexidade do CIM na sua integralidade, que deve envolver questões tão diversas quanto o planejamento, o projeto, a construção, a operação e a manutenção dos ativos urbanos, bem com a expansão e a renovação dos mesmos, considerando todas as suas complexidades.

Assim, buscando contribuir para estabelecimento de uma conceituação mais abrangente e precisa para o paradigma City Information Modeling, no contexto deste trabalho a discussão sobre o CIM se dará a partir de duas premissas que permeiam a literatura que trata da questão. A primeira estabelece que City Information Modeling (CIM) é uma extensão ou uma analogia do conceito de Building Information Modeling (BIM) aplicado às cidades e aos seus processos. A segunda, decorrente da primeira, considera que o CIM está para a cidade assim como o BIM está para a edificação.

Contribuindo para a sedimentação terminológica sobre o paradigma $\mathrm{CIM}$, foi proposto e tem-se firmado o termo na língua portuguesa, Modelagem da Informação da Cidade, como uma analogia terminológica ao termo Modelagem da Informação da Construção, como também apontam Corrêa e Santos (2015), e Amorim (2015). Cabe lembrar que o termo Modelagem da Informação da Construção foi definido e adotado pela Associação Brasileira de Normas Técnicas (ABNT) como a tradução para Building Information Modeling (BIM).

Postas essa premissas, a discussão avança calcada nesses pressupostos, no sentido do estabelecimento de alguns requisitos que possam convergir para a implementação de sistemas e ferramentas que viabilizem num futuro próximo a Modelagem da Informação da Cidade. Analogamente ao BIM, nesse processo pelo menos três aspectos devem ser observados: 
- a necessidade de formular o conceito de CIM de forma clara e abrangente e de harmoniza-lo com diversos outros conceitos correlatos;

- a imprescindível caracterização dos diversos processos envolvidos na cidade e por conseguinte a definição dos requisitos a serem atendidos pelo modelo CIM, e

- o estabelecimento de modelos e padrões para a implementação do "modelo CIM", bem como o desenvolvimento das ferramentas que lhe darão o suporte operacional.

\section{O papel do CIM como indutor da cidade inteligente}

Como pode ser visto, Cidades Inteligentes e CIM não correspondem a um mesmo conceito, ou a uma mesma aplicação, mas sim, a conceitos e aplicações diversas que podem ser consideradas como complementares.

Pode-se resumir em linhas gerais que, enquanto a Smart City tem foco na qualidade de vida, no exercício da cidadania e no uso racional dos recursos e meios de produção, o foco do CIM está no planejamento, no projeto, na construção, na gestão (operação e manutenção) e no monitoramento da cidade, ou seja, naquilo que se poderia chamar de infraestrutura de uma "cidade Inteligente".

Nesse sentido, o paradigma CIM pode ser entendido como uma plataforma tecnológica (software, equipamentos e processos), apoiada por recursos humanos e conceitos das várias áreas de aplicação, perfeitamente integrados para suportar a infraestrutura da cidade.

No que pese a discussão sobre City Information Modeling esteja cada vez mais presente na literatura técnica e nos diversos fóruns de debates, nem mesmo a sua conceituação está plenamente estabelecida, como citado anteriormente.

Por outro lado, como observado, grande parte do aparato tecnológico necessário à implementação do CIM já existe sob a forma de ferramentas para uso stand alone, voltadas à solução de problemas específicos.

Cabe ressaltar que uma parte desta tecnologia já está incorporada nas plataformas para Building Information Modeling, como as ferramentas para planejamento e gestão da construção, orçamentação etc., e que não são especificas para a aplicação em edificações. Deste modo, com a introdução de pequenos acréscimos nos seus códigos fontes e estruturas de dados, essas ferramentas poderão ter os seus escopos expandidos e serem facilmente integradas às ferramentas CIM.

Assim, a principal tarefa que se coloca é o estabelecimento de uma visão integradora e colaborativa como é necessária às aplicações CIM, o que de uma forma mais direta se traduz na capacidade de colaboração entre os vários agentes urbanos, na interoperabilidade dos sistemas fundamentados nas Tecnologias de Informação e Comunicação, em interfaces comuns, intuitivas e amigáveis para as ferramentas, dentre outros aspectos igualmente relevantes.

Finalmente, outro aspecto essencial para a viabilização do CIM já esta sendo encaminhado, que é a criação e a adoção de padrões internacionais, abertos, compartilháveis para o armazenamento e o fluxo de informações, de modo que o setor de Arquitetura, Engenharia, Construção (AECO), alcancem práticas mais sustentáveis, eficientes e colaborativas de trabalho ao longo de todo ciclo de vida dos empreendimentos.

Através de iniciativa do Open Geospatial Consortium (OGC, 2012), foi estabelecido o padrão CityGML, que é um formato de dados para a representação numérica tridimensional de elementos urbanos, visando a implementação de aplicações 3D em Sistema de Informações Geográficas. Padrão este já largamente empregado na Europa e que foi usado para a confecção do modelo de várias cidades.

Outra iniciativa importante vem da buildingSMART, que anuncia para o futuro release do Industry Foundation Classes, O IFC 5, a capacidade de representar os principais elementos da infraestrutura da cidade. Essa promessa ganha credibilidade visto que a atual versão (IFC4), aprovada em 2013 e em uso a partir de 2014, já é um padrão internacional definido pela ISO (ISO 16739:2013), conforme Liebich (2013), Liebich (s. d.), e buldingSmart (2015).

Assim, considera-se que estas duas realidades materializadas por dois padrões internacionais para representação de dados urbanos no contexto do Geographic Information Systems (GIS) e do Building Information Modeling (BIM), deverão convergir visando a total compatibilidade entre eles nos próximos anos, representam o embrião para as estruturas de dados e as plataformas de software que viabilizarão as futuras aplicações de Modelagem da Informação da Cidade através das ferramentas CIM de primeira geração.

Todas estas características propiciadas pela Modelagem da Informação da Cidade, aliadas à base de informações já existentes nas diversas áreas de conhecimento que concorrem para as aplicações urbanas, apresentam um potencial para mudar a forma de conceber, planejar, projetar, construir e operar as cidades, tornando-as mais adequadas, eficientes e seguras para os seus habitantes.

Nesse sentido, a adoção da Modelagem da Informação da Cidade, pode ser uma estratégia para que a mesma possa melhorar a sua infraestrutura e os serviços prestados aos seus habitantes, visando alcançar o status de "Cidade Inteligente", se for considerado que a implantação do CIM, visa a eficiência e eficácia dos sistemas de infraestrutura urbana, e como consequência dos vários serviços e atividades dependentes desses sistemas de infraestrutura. 
Assim, para se atingir o status de Cidade Inteligente, é essencial que todos os serviços urbanos sejam da mais alta qualidade, visto que o simples emprego dos aplicativos e abordagens para Smart Cities, baseados nas Tecnologias de Informação e Comunicação, não possuem o poder de transformar a realidade, muito pelo contrário, sem a infraestrutura necessária, essas tecnologias apenas evidenciam o baixo nível de desenvolvimento urbano e a precariedade dos serviços prestados.

A adoção da Modelagem da Informação da Cidade e preparo da mesma para atingir o status de Cidade Inteligente são dois empreendimentos de grande envergadura, tanto pelos custos e riscos tecnológicos envolvidos, quanto pelas dificuldades operacionais, administrativas e legais. Entretanto, esses sistemas são dotados de uma relativa interdependência, e de uma grande afinidade, sendo capazes de produzirem sinergia, na medida em que toda a cidade é minuciosamente analisada e muitas das soluções tecnológicas a serem adotadas poderão beneficiar a ambas iniciativas.

Por fim, a decisão da implementação integrada entre City Information Modeling (CIM) e Smart City reduz os riscos inerentes aos processos de implantação, minimiza os custos e maximiza os resultados.

\section{Considerações finais}

Como discutido, os desafios para as cidades do século XXI estão colocados e são preocupantes, seja pelo seu tamanho, complexidade e quantidade de recursos financeiros e tecnológicos para a sua superação, seja ainda pelo curto prazo em que as medidas são necessárias.

Deste modo, faz-se necessário o desenvolvimento de abordagens como as Cidades Inteligentes e a Modelagem da Informação da Cidade como uma estratégia para que os problemas possam ser encaminhados de forma mais adequada.

O equacionamento das questões aqui colocadas contempla sem dúvida a necessidade de tecnologias especificas, que certamente não ainda totalmente disponíveis, e que demandarão um significativo esforço de desenvolvimento por parte das softhouses nos próximos anos.

Também o desafios de se produzir os modelos de dados capazes de atender às demandas dessas aplicações, também já estão colocados, e esforços estão sendo feitos pela buildingSmart Community, e pela Open Geospatial Consortium, através do desenvolvimento dos padrões IFC e CityGML, respectivamente.

Por fim, ressalta-se que aspectos como a formação de pessoal técnico para lidar com os desafios das cidades do século XXI, de forma mais integrada, colaborativa e compartilhando uma base de dados única, é uma questão que ainda não foi olhada com a devida atenção pelas administrações municipais, e que é tão importante quanto as demais questões aqui citadas, e que, em última instância são determinantes para o sucesso das implementações visando as cidades Inteligentes e o CIM.

\section{Agradecimentos}

Ao Conselho Nacional de Desenvolvimento Científico e Tecnológico (CNPq), pela concessão de bolsa de pósdoutorado e de produtividade em pesquisa.

\section{Referências}

ONU. (2014). World Urbanization Prospects. The 2014 Revision Highlights. United Nations, 2014.

Amorim, A. L. (2015). Discutindo City Information Modeling (CIM) e conceitos correlatos. Gestão e Tecnologia de Projetos, São Paulo, v. 10, n. 2, p. 87-99, jul./dez. 2015. Doi: http://dx.doi.org.br/10.11606/gtp.v10i2.103163

Bott, Helmut. Digital Planning Systems: designing the future sustainable city. Disponível em: <http://www.eservicessa.org/event/uploadfiles/pdf/1_digit_plan_systems_endf.pdf>. Acesso em: 19 maio 2016.

Lee, J. \& Hancock, M. Toward a framework for Smart Cities: a comparison of Seoul, San Francisco \& Amsterdam. Standford Program on Regions of Innovation and Entrepreneurship, 2012.

Finguerut, S.; Fernandes, J. M. (2014). Planejando as cidades do século XXI. Cadernos FGV Projetos, Rio de Janeiro, ano 10, n. 24, p. 30-39.

Gil, J.; Beirão, J.; Montenegro, N. \& Duarte, J. Assessing Computational Tools for Urban Design: towards a city information model. In: EDUCATION AND RESEARCH IN COMPUTER AIDED ARCHITECTURAL DESIGN IN EUROPE, 28., 2010, Prague. Proceedings... Prague, Czech Technical University in Prague / Faculty of Architecture, 2010.

HISHAM, Ahmad. The new trend of CIM. In: Ahmad's Findings. Disponível em: <http://ahmadsfindings.blogspot.de/2010/05/newtrend-of-cim.html>. Acesso em: 19 abr. 2015.

GIL, J.; ALMEIDA, J.; DUARTE, J. The backbone of a City Information Model (CIM): Implementing a spatial data model for urban design. In: EDUCATION AND RESEARCH IN COMPUTER AIDED ARCHITECTURAL DESIGN IN EUROPE, 29., 2011, Ljubljana. Proceedings... Ljubljana, University of Ljubljana / Faculty of Architecture, 2011.

STOJANOVSKI, T. City Information Modeling (CIM) and Urbanism: blocks, connections, territories, people and situations. In: SYMPOSIUM ON SIMULATION FOR ARCHITECTURE AND URBAN DESIGN, 2013, San Diego. Proceedings... San Diego: ACM, 2013.

SCHIEFELBEIN, J. et al. Development of a City Information Model to support data management and analysis of building energy systems within complex city districts. In: CISBAT 2015 INTERNATIONAL CONFERENCE FUTURE BUILDINGS AND DISTRICTS: SUSTAINABILITY FROM NANO TO URBAN SCALE, X., 2015, Lausanne. Proceedings... Disponível em: <https://www.semanticscholar.org/paper/Development-of-a-CityInformation-Model-to-Support-Schiefelbein-Javadi/ 40f97b132b790298e36a9bfd5c48c2a9b47d21e2/pdf>. Acesso em: 24 maio 2016.

Corrêa, F. R. \& Santos, E. T. Na direção de uma modelagem da informação da cidade (CIM). In: ENCONTRO BRASILEIRO DE TECNOLOGIA DE INFORMAÇÃO E COMUNICAÇÃO NA 
CONSTRUÇÃO, 7., 2015, Recife. Anais... Porto Alegre: ANTAC, 2015.

OPEN GEOSPATIAL CONSORTIUM (OGC). OGC City Geography Markup Language (CityGML) Encoding Standard. Editores: Gerhard Gröger, Thomas H. Kolbe, Claus Nagel, Karl-Heinz Häfele. Versão: 2.0.0. OCG, 2012.

Liebich, T. IFC for Infrastructure - Separation between BIM and GIS requirements. buildingSMART, (s. d.). Disponível em: <http://www.iowadot.gov/bridge/3D/Presentations/

20121017_IFC_for_Infrastructure.pdf>. Acesso em: 7 abr. 2016.

Liebich, T. IFC4 - the new buildingSMART Standard: What's new on IFC4. buildingSMART, 2013. Disponível em: <http://www.buildingsmart-tech.org/specifications/ifcreleases/ifc4-release/buildingSMART_IFC4_Whatisnew.pdf>. Acesso em: 7 abr. 2016.

BUILDINGSAMRT. IFC Alignment Project, Process Map and Use Cases (informative). BuilgingSmart, 2015. Disponível em: <http://www.buildingsmart-tech.org/downloads/ifc/ifc5-extensionprojects/ifc-alignment/ifc-alignment-exchange-scenarios-fs $>$. Acesso em: 20 maio 2016. 